Results: Waist/height ratio $(\mathrm{W} / \mathrm{h})$ is greater in $\mathrm{M}$ general sample $(P=0 \cdot 01)$ but also in POST-F:PRE-F $(P=0 \cdot 05)$ and in PRE-M:PRE-F $(P=0 \cdot 0001)$. At large, blood glucose was higher in $\mathrm{M}$, independently from puberty $(P=0 \cdot 01)$, while insulin was similar in $\mathrm{F} / \mathrm{M}$. After sub-grouping, insulin was higher in post-F/M (both $P=0 \cdot 0001) v$. PRE, while glucose was higher in POST-F:POST-M $(P=0 \cdot 01)$.

Similar behaviour for insulin resistance-homeostasis model assessment (IR-HOMA): higher in POST-F/M $v$. PRE (both $P=0 \cdot 0001$ ). Besides, IR-HOMA $>2.5$ risk is higher in POST (whole sample, F, M), but POST-M have a greater risk $(\mathrm{OR}=2 \cdot 11$ POST:PRE, $P=0 \cdot 0001 ; \mathrm{OR}=2 \cdot 45$ POST-M:PRE-M, $\quad P=0 \cdot 02 ; \quad$ OR $=1.94 \quad$ POST-F:PRE-F, $P=0 \cdot 01)$.

Conclusions: $\mathrm{M}$ attending our outpatients service seems in poorer nutritional (higher $\mathrm{W} / \mathrm{h}$ ) and metabolic conditions (higher pathologic IR-HOMA risk) than F, with a slight indication that abdominal fat distribution might not be the only explanation for IR appearance: other factors should be considered and studied.

\title{
29 - Insulin resistance risk among ex-preterm overweight/obese patients
}

\author{
A Piedimonte, A Mosca, AM Caiazzo, D Guttadoro, A Cafarotti, CM L'Amante, R Mercurio, \\ RE Papa and A Vania
}

Centro di Dietologia e Nutrizione Pediatrica, I Facoltà di Medicina e Chirurgia, 'Sapienza' Università di Roma, Italy

Introduction: According to the 'thrifty phenotype' hypothesis, ex-preterm (ExP) children, if overfed in infancy, show a greater insulin resistance (IR) risk than AGA (appropriate gestational age) children. ExP also shows a larger waist circumference (W), due to greater extent of abdominal fat: this might trigger off IR.

Method: 655 valid overweight/obese patients (F 356, M 309 ; average age $=10 \cdot 43(\mathrm{SD} 2 \cdot 84)$ years) were considered: ExP 118 (F 62, M 56), AGA 547 (F 294, M 253). Anthropometric indexes studied were $\mathrm{W}$ and waist/height ratio (W/h). Insulin resistance-homeostasis model assessment (IR-HOMA), studied in 569 patients (ExP 102: F 54, M 48; AGA 467: F 254, M 213), led to sub-grouping them in: IR-HOMA >2.5 (ExP 53: F 26, M 27; AGA 226:
F 129, M 97) and IR-HOMA <2.5 (ExP 49: F 28, M 21; AGA 241: F 125, M 116). Statistical analysis used Student's $t$ and $\chi^{2}$ tests.

Results: W was $>95$ thC in $97 \cdot 4 \%$ of $\operatorname{ExP} v \cdot 91 \cdot 4 \%$ of AGA; W/h was pathologic $(>0.5)$ in $92.4 \%$ of $\operatorname{ExP} v$. $89 \cdot 0 \%$ of AGA. ExP have a higher risk of $\mathrm{W}>95$ thC and $\mathrm{W} / \mathrm{h}>0.5$ than AGA (W OR: $\mathrm{F}=5 \cdot 33, \mathrm{M}=2 \cdot 23$; W/h $\mathrm{OR}=1.5$ in both $\mathrm{F} / \mathrm{M}$, respectively). ExP also have IRHOMA $>2.5$ more frequently, with higher risk for M (OR: $\mathrm{F}=0 \cdot 9 ; \mathrm{M}=1 \cdot 5$ ).

Conclusions: In our experience, ExP of both genders show a greater extent of $\mathrm{W}>95^{\circ} \mathrm{C}$ and $\mathrm{W} / \mathrm{h}>0.5$ than AGA, but an IR risk just slightly higher $(\mathrm{OR}=1 \cdot 25)$. $\mathrm{M}$ ExP seems to be at higher risk than F: literature lacks of data about this point.

\section{0 - Relationship among insulin resistance, blood lipids and blood pressure in a population of paediatric overweight/obese patients}

\author{
A Mosca, A Piedimonte, AM Caiazzo, RE Papa, D Guttadoro, A Grimaldi, \\ S Zorkadis, A Vania \\ Centro di Dietologia e Nutrizione Pediatrica, I Facoltà di Medicina e Chirurgia, 'Sapienza' Università di Roma, Italy
}

Introduction: Within the ample debate about Metabolic Syndrome, its primum movens and its pathophysiology, a relationship among high insulin resistance-homeostasis model assessment (IR-HOMA), blood lipids (BL: triglycerides 
TG, total cholesterol CHOL, LDL-CHOL) and blood pressure (BP) in both genders is well known. Some authors (see Sinalko 2004) even documented a constant, progressive increase of $\mathrm{BL}$ and $\mathrm{BP}$, proportional to IRHOMA values.

Method: 288 patients with IR-HOMA $>2 \cdot 5$ (F 160, M 128), out of 683 overweight/obese ones, aged 9-14 years (average age $=11.09$ (SD 2.62) years) were divided into seven IR-HOMA and one unit stepped groups. Every group's BL and BP were confronted with the groups around and with the opposite gender's corresponding group. Statistical analysis: Student's $t$ test and parametric/ non-parametric correlation tests.

Results: In both genders TG decrease $(P<0.05)$ in IRHOMA groups $1-3$, then increase $(P<0 \cdot 05)$; F/M CHOL increases only in groups 6 and $7(P<0 \cdot 05)$. Among $\mathrm{F}$,
LDL-CHOL $(P<0 \cdot 05)$ and $\mathrm{BP}(P<0 \cdot 01)$ also increase in groups 6 and 7 , while among M, LDL-CHOL is increased only in some IR-HOMA groups $(1,3,6)$ and BP increases in group 5 (all $P<0.05$ ). Pairing opposite gender groups, $\mathrm{BL}$ and BP differences are significant in single groups, not at large. Parametric/non-parametric correlations were non-significant.

Conclusions: Notwithstanding our large data allow a quite accurate comparison of metabolic parameters, a progressive, much less constant, increase of any BL or $\mathrm{BP}$, proportional to IR-HOMA increase, could not be demonstrated. Our contribution outlines once more that BL, BP and IR-HOMA are undoubtedly bound, but more factors that IR alone (like genetics, overweight degree and hyperhomocysteinemia) presumably influence this link.

\title{
31 - C-reactive protein: a marker of adiposity or cardiometabolic comorbidities of paediatric obesity?
}

\author{
Andreia Teles ${ }^{1}$, Carla Rego ${ }^{1,2}$, Claudia Dias $^{3}$ and Joao-Tiago Guimaraes ${ }^{4}$
}

${ }^{1}$ Nutrition Unit, Pediatrics Department \& Clinical Pathology Department, Hospital S. Joao, University of Porto, Portugal: ${ }^{2}$ Pediatrics Department, University of Porto, Portugal: ${ }^{3}$ Biostatistics and Medical Informatics (CINTESIS), University of Porto, Portugal: ${ }^{4}$ Biochemistry Department, Faculty of Medicine, University of Porto, Portugal

Background/aims: Childhood obesity is a public health problem. The association between obesity and low-grade inflammation is well established. Our aim is to evaluate the association between C-reactive protein (CRP) and cardiometabolic comorbidities in paediatric obesity.

Material and method: Obese children/adolescents with nutritional obesity followed in our outpatient clinic ( $n$ 354) were included. Duration of disease (years), BMI $Z$ score (Center for Disease Control), percentage of fat mass (dual energy X-ray absorptiometry) and waist circumference were evaluated. Blood pressure, lipid profile and CRP were measured and homeostasis model assessment-insulin resistance (HOMA-IR) was calculated.

Results: The mean chronological age was $10 \cdot 1$ years (SD $3 \cdot 2 ; \min =1 \cdot 7 ; \max =16 \cdot 9)$ with no differences between gender. Same data related to descriptive analyses can be observed in Table 1. CRP was positive and significantly correlated with BMI $Z$-score $(r=0 \cdot 271 ; P<0 \cdot 001)$, \%fat mass $(r=0.366 ; P<0.001)$ and waist circumference $(r=0 \cdot 198 ; P<0 \cdot 001)$. A strong positive correlation was observed between CRP and fat mass, even for short duration of disease $(<2$ years: $r=0.731 ; P<0 \cdot 001)$. No correlations were observed between CRP and lipid profile variables (total, HDL- and LDL-cholesterol, Apo lipoproteins $\mathrm{A} 1$ and $\mathrm{B}$ and triglycerides), systolic and diastolic blood pressure and HOMA-IR, independently of duration of disease.

Conclusions: Magnitude of obesity and adiposity as also intraabdominal fat deposition are predictors of early expression of low-grade inflammation. CRP seems not to be a sensitive/early marker of cardiometabolic comorbidity of paediatric obesity.

\begin{tabular}{|c|c|c|c|c|c|c|c|}
\hline & \multicolumn{2}{|c|}{ Total ( $n$ 354) } & \multicolumn{2}{|c|}{ Females ( $n$ 182) } & \multicolumn{2}{|c|}{ Males ( $n$ 172) } & \multirow[b]{2}{*}{$P$} \\
\hline & Mean & SD & Mean & SD & Mean & SD & \\
\hline BMI Z-score & $4 \cdot 1$ & $1 \cdot 7$ & $4 \cdot 0$ & $1 \cdot 7$ & $4 \cdot 2$ & $1 \cdot 8$ & 0.465 \\
\hline Waist (\%90th Pc) & $117 \cdot 7$ & $12 \cdot 4$ & $118 \cdot 2$ & $15 \cdot 9$ & $116 \cdot 4$ & $11 \cdot 4$ & 0.076 \\
\hline$\%$ Fat mass - (DXA) & $45 \cdot 8$ & $6 \cdot 1$ & $47 \cdot 2$ & $5 \cdot 7$ & $44 \cdot 3$ & $6 \cdot 2$ & 0.002 \\
\hline CRP & $0 \cdot 31$ & $0 \cdot 4$ & 0.32 & 0.4 & 0.31 & $0 \cdot 4$ & 0.581 \\
\hline
\end{tabular}

CRP, C-reactive protein. 